\title{
In Silico Blood Glucose Control for Type 1 Diabetes with Meal Announcement Using Carbohydrate Intake and Glycemic Index
}

\author{
Claudia Cecilia Yамамото Noguchi, ${ }^{*, \# ~ S h o g o ~ H a s h i m o t o, ~}{ }^{*}$ Eiko Furutani ${ }^{*}$
}

\begin{abstract}
An increasing number of closed-loop blood glucose (BG) control algorithms have been developed in recent years with the 'artificial pancreas' as the ultimate goal, although tight postprandial BG control remains an elusive goal. In this report, the authors propose a novel semi closed-loop BG control algorithm with meal announcement, which involves computation of the optimal continuous subcutaneous insulin infusion for a specific meal $60 \mathrm{~min}$ prior to mealtime. It utilizes a mathematical model of glucose-insulin metabolism to predict the impact of carbohydrates on postprandial BG levels based on carbohydrate intake and glycemic index (GI) value. The optimal pre-meal insulin is infused until mealtime, after which the control algorithm switches to model predictive control (MPC) to stabilize postprandial glycemia at the target value of $100 \mathrm{mg} / \mathrm{dL}$ (5.55 mmol/L). In silico results for four representative foods with GI values spanning a wide range show that in the case of exact patient-model match with precise information of carbohydrate composition and mealtime, postprandial BG levels can be maintained between 86$134 \mathrm{mg} / \mathrm{dL}(4.78-7.44 \mathrm{mmol} / \mathrm{L})$ and $86-152 \mathrm{mg} / \mathrm{dL}$ (4.78-8.44 mmol/L) for $50 \mathrm{~g}$ and $100 \mathrm{~g}$ of carbohydrates, respectively. With consideration of intra-patient variability and meal-related uncertainties regarding the estimated carbohydrate amount and start of meal consumption, the BG control range is $75-159 \mathrm{mg} / \mathrm{dL}(4.17-8.83 \mathrm{mmol} / \mathrm{L})$ with no critical hypoglycemic episodes.
\end{abstract}

Keywords: glycemic index, model predictive control, prandial state, tight blood glucose control, type 1 diabetes.

Adv Biomed Eng. 5: pp. 124-131, 2016.

\section{Introduction}

Type 1 diabetes (T1D) is one of several forms of diabetes caused specifically by an abrupt decline in endogenous insulin secretion from pancreatic $\beta$-cells, resulting in extremely high blood glucose (BG) levels. To prevent life-threatening complications in the long term, T1D patients must undergo a basal-bolus insulin therapy that requires an accurate estimation of the single-bolus insulin dose based on the total carbohydrate intake at every single meal. Despite continuous development of correction factors to increase the accuracy of estimation of prandial insulin needs [1], T1D patients still not only suffer from postprandial hyper- and/or hypoglycemic episodes-with their respective accompanying symptoms-due to insulin bolus miscalculation, but also are constantly exposed to wide post-meal glycemic excursions that compromise endothelial function and directly impacts microvascular complications [2]. Thus postprandial hyperglycemia has been recently acknowledged to have a more deleterious effect on the overall management of diabetes than fasting BG levels [3].

To reduce the burden of T1D treatment, there has been an increasing number of studies on automatic BG control systems aiming at the development of the 'artificial pancreas' [4]. Proportional-integral-derivative control [5] and model predictive control (MPC) [6] are the most widely utilized algorithms for BG control purposes, with different approaches being constantly proposed,

Received on March 30, 2016; revised on September 1, 2016; accepted on September 28, 2016.

* Department of Electrical Engineering, Kyoto University, Kyoto, Japan.

\# Kyoto University Katsura, Kyoto 615-8510, Kyoto, Japan.

E-mail: ccyamamoto@ rotary.kuee.kyoto-u.ac.jp including fully closed-loop control algorithms with automatic meal detection based on changes in BG levels [7] and semi closed-loop systems with improved postprandial BG control performance at the expense of additional input from the patient for an intended meal (meal announcement) [8]. Others favor a fixed pre-meal bolus of 2 IU [9] or over-bolus [10] along with closedloop BG control, while a recent intraperitoneal insulin infusion [11] offers an interesting alternative to subcutaneous route due to faster insulin responses. Although these approaches in diurnal BG control have been shown to maintain postprandial BG excursion largely within a specific range, they have not achieved the target maximum peak BG levels below $180 \mathrm{mg} / \mathrm{dL}$ (10.0 mmol/L) recommended by the American Diabetes Association (ADA) for prandial BG management in T1D patients [12]. These previous clinical results seem to indicate that tight diurnal BG control might be an unattainable goal, which is arguably attributed to the great plasma glucose appearance from carbohydrate-rich meals, combined with slow insulin absorption kinetics and delayed effect on BG levels of subcutaneous administration by up to several times compared to normal endogenous insulin secretion in nondiabetics.

Based on observation in clinical studies in which insulin bolus given 15 min prior to meal consumption reduced postprandial glycemic excursion [13], the authors previously developed a prandial control algorithm that satisfactorily maintained postprandial BG levels within the recommended clinical range, by combining a pre-meal single insulin bolus with postprandial closed-loop BG control initiated at mealtime [14] to compensate for the rapid and large glycemic increase after a meal. Such BG control algorithm utilizes a novel mathematical model of glucose-insulin metabolism in T1D [15, 16], which uses the amount of carbohydrates as well as the glycemic index (GI) [17] to accurately predict the glucose absorption rate according to the type of 
carbohydrate consumed and the impact on postprandial BG level.

Because of the limited performance of previous BG control algorithms during postprandial state, the present study seeks to determine the degree of attainability of tight prandial BG control in T1D in silico. In this way, provided meal announcement information of carbohydrate amount and GI value of the intended meal, the optimal continuous subcutaneous insulin infusion is determined from an insulin optimization problem computed at the initial time, and accordingly infused to the patient until mealtime. Thence, closed-loop MPC control is used for the following 440 min (> 7 hours) post-meal to ensure complete stabilization of BG level at the target value of $100 \mathrm{mg} / \mathrm{dL}$ (5.55 mmol/L) for the assessment of the BG control performance for different GI foods, as well as patient and food-related uncertainties.

The present article includes an elaborate description of the prandial BG control algorithm in Section 2, an in silico evaluation of BG control performance in Section 3, followed by further discussions of the results in Section 4, and conclusions in Section 5.

\section{Method}

\subsection{Blood Glucose Control Strategy}

In the present study, we use discrete-time control systems with sampling period $\Delta t$ and discrete time step $k$, where $t=k \Delta t$. Utilizing the mathematical model developed in a previous study (see supplementary material) [15, 16], the novel control algorithm proposed in the present section is detailed as follows. First, let $k_{\text {food }}$ be defined as mealtime for the T1D patient, where $k_{\text {food }}=0$ is the initially intended mealtime. From this, let $k_{i}(<0)$ be defined as the initial time of prandial BG control and potential start of preprandial insulin infusion; and let $k_{f}(>0)$ be the ending time in prandial BG control at which BG levels are completely stabilized at the target value (Fig. 1). The purpose of the present control is to maintain prandial BG levels within those recommended by the ADA (Table 1), Provided that $k_{\text {food }}=0$, the control strategy in the present study is divided into two parts. Initially, optimal prepran-

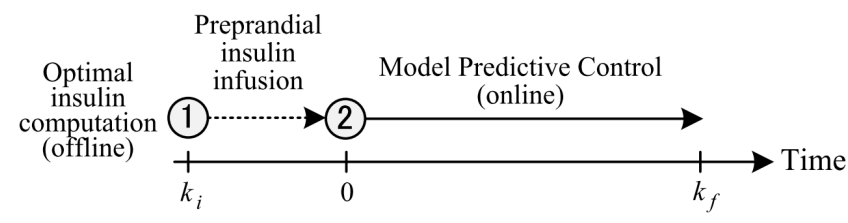

Fig. 1 Insulin infusion strategy for the prandial BG control algorithm in the present study. (1) At $k=k_{i}$, optimal prandial insulin infusion from $k=k_{i}$ to $k_{f}$ is calculated from meal content information (amount and type of carbohydrate), and infused accordingly from $k_{i}$ to 0 (mealtime). (2) At $k=0$, the control algorithm switches to MPC to maintain BG levels at the target value.

Table 1 ADA recommendations for BG management in T1D [12].

\begin{tabular}{lc}
\hline \hline Condition & BG levels [mg/dL] \\
\hline Fasting / premeal & $70-130$ \\
Peak postprandial & $<180$ \\
Premeal / overnight (pregnancy) & $60-99$ \\
Peak postprandial (pregnancy) & $100-129$ \\
\hline \hline
\end{tabular}

dial insulin infusion to maintain tight BG control throughout the prandial state from $k=k_{i}$ to $k_{f}$ is computed at $k=k_{i}$, although insulin is effectively infused only from $k=k_{i}$ to 0 (mealtime). Thereafter, the control algorithm automatically switches to closed-loop BG control utilizing MPC for stabilization and maintenance of BG levels at the target BG value until the final time of $k=k_{f}$. At mealtime, a single-meal is represented by an impulse signal in the input $G_{\text {food }}(t)$ with specific carbohydrate intake in the input of the T1D patient model given by Eq. (S2) in the supplementary material.

\subsection{Prandial Insulin Optimization}

At the initial time $k_{i}$, the optimal prandial insulin infusion is calculated by the cost function given by

$$
J_{\mathrm{opt}}=\sum_{i=0}^{k_{f}-k_{i}} Q(E) E^{2}\left(k_{i}+i \mid k_{i}\right)+\sum_{i=k_{i}}^{k_{f}} R \Delta u_{\mathrm{s}}^{2}(i)
$$

where $E\left(k_{i}+i \mid k_{i}\right)=\hat{y}\left(k_{i}+i \mid k_{i}\right)-W_{\mathrm{R}}$ is the deviation of the estimated model output $\hat{y}\left(k_{i}+i \mid k_{i}\right)$ from the target BG value $W_{\mathrm{R}} ; \hat{y}\left(k_{i}\right.$ $\left.+i \mid k_{i}\right)$ is the model-predicted BG value as the output $\hat{y}(k)=$ $G(k \Delta t)$ at $k=k_{i}+i$ provided the initial condition $\hat{y}\left(k_{i}\right)$ of subcutaneous insulin infusion sequence $u_{\mathrm{s}}(i)$ as given in Eq. (S10); $\Delta u_{\mathrm{s}}(i)=u_{\mathrm{s}}(i)-u_{\mathrm{s}}(i-1)$ is the change in insulin rate; and $Q$ and $R$ are weighting matrices for $E$ and $\Delta u_{\mathrm{s}}(i)$, respectively. In particular, we consider $\Delta u_{\mathrm{s}}(i)$ to be more suitable than $u_{\mathrm{s}}(i)$ in the cost function $J_{\text {opt }}$ to provide a smooth continuous variable insulin infusion and eliminate steady-state offset errors. Moreover, as a countermeasure of critical hypoglycemia, we consider a variable weighting matrix $Q$ depending on the BG deviation $E$, with a larger weight $Q_{\mathrm{LO}}>Q$ for values below the reference BG given as

$$
Q(E)=\left\{\begin{array}{ll}
Q, & E \geq 0 \\
Q_{\mathrm{LO}}, & E<0
\end{array} .\right.
$$

The prandial optimization problem is thus

$$
\begin{array}{ll}
\underset{\left\{u_{\mathrm{s}}(k)\right\}}{\operatorname{minimize}} & J_{\mathrm{opt}} \\
\text { subject to } & 0 \leq u_{\mathrm{s}}(k) \leq U_{\text {pre }}, \quad k<0 \\
& 0 \leq u_{\mathrm{s}}(k) \leq U_{\text {post }}, \quad k \geq 0
\end{array}
$$

where $U_{\text {pre }}$ and $U_{\text {post }}$ are the maximum preprandial and postprandial insulin infusions, respectively, allowed for patient safety to avoid over-insulinization. Note that although the optimal insulin for $0 \leq k \leq k_{f}$ is also computed in Eq. (3), only preprandial bolus insulin from $k_{i}$ to 0 is actually infused to the patient.

\subsection{Postprandial BG control algorithm}

After the total optimal insulin is computed according to Eq. (3) and effectively infused to the T1D patient until $k=0$, the control algorithm switches to closed-loop BG control based on an MPC algorithm with cost function given by

$$
J_{\mathrm{MPC}}(k)=\sum_{i=N_{1}}^{N_{2}} Q(e) e^{2}(k+i \mid k)+\sum_{i=0}^{N_{u}-1} R \Delta u_{\mathrm{S}}^{2}(k+i)
$$

where the weighting matrix $Q(e)$ also follows Eq. (2). $N_{1}$ and $N_{2}$ define the prediction horizon; $N_{\mathrm{u}}$ is the control horizon; and $e(k+$ $i \mid k)=\hat{y}(k+i \mid k)-w(k+i \mid k)$ is BG deviation of the model prediction from the reference trajectory given by

$$
w(k+i \mid k)=\alpha^{i} w(k \mid k)+\left(1-\alpha^{i}\right) W_{\mathrm{R}},
$$

with convergence rate constant $0<\alpha<1$ and reference trajectory span of $i=N_{1}, \ldots, N_{2}$. In particular, the reference trajectory in Eq. (5) is an exponential curve for smoother approach of BG to- 
wards the target $\mathrm{BG}$ value $W_{\mathrm{R}}$, to prevent abrupt variations in $\mathrm{BG}$ level throughout the postprandial period.

The MPC optimization problem to solve is thus

$$
\begin{array}{ll}
\underset{\left\{u_{\mathrm{s}}(k+i)\right\}}{\operatorname{minimize}} & J_{\mathrm{MPC}}(k) \\
\text { subject to } & 0 \leq u_{\mathrm{s}}(k+i) \leq U_{\text {post }}, \quad 0 \leq i \leq N_{\mathrm{u}}-1
\end{array}
$$

from which the optimal postprandial insulin infusion is iteratively computed by evaluation of $J_{\mathrm{MPC}}(k)$ and respective insulin infusion to the patient at each step from $k=0$ until the end of the prandial state at $k=k_{f}$.

\subsection{Clinically relevant control parameters}

Parameter settings of the present control algorithm take into consideration clinical requirements for BG control application. Due to the rapid BG fluctuation from meal-derived glucose appearance, we consider $\Delta t=1 \mathrm{~min}$ adequate as commonly used in clinical studies [7, 18]. Start of preprandial insulin infusion is set at $k_{i}=-60$ to provide sufficient margin for the computation of the optimal preprandial insulin infusion and $k_{f}=440$ to guarantee complete stabilization of late postprandial BG levels. Target BG level is set at $W_{\mathrm{R}}=100 \mathrm{mg} / \mathrm{dL}(5.55 \mathrm{mmol} / \mathrm{L})$ according to the ADA clinical recommendations. To prevent insulin overdosing, maximum preprandial insulin infusion rate is set at $U_{\text {pre }}=2 \mathrm{IU} /$ min considering that the insulin bolus required (including differences in insulin-to-carbohydrate ratio) for a regular meal ranges from 3.7 to 10.9 IU [19], whereas maximum postprandial insulin infusion rate is set at $U_{\text {post }}=0.05 \mathrm{IU} / \mathrm{min}$, which although considerably above basal profiles [20], is considered adequate since it includes not only basal insulin needs but also residual prandial insulin needs, especially for foods with low GI. Tuning parameters for the prandial control algorithm are $Q=1, Q_{\mathrm{LO}}=10, R=$ $111, N_{1}=40$ steps, $N_{2}=130$ steps, $N_{\mathrm{u}}=1$, and $\alpha=0.995$, such that the control algorithm satisfies the ADA clinical recommendations for prandial BG management (Table 1). In particular, the values of $N_{1}$ and $N_{2}$ are set to include the maximum hypoglycemic effect of insulin, which occurs between 1-2 hours after infusion, and the unitary value in $N_{\mathrm{u}}$ for increased robustness in the control system against errors [21].

\subsection{Simulation}

In order to assess the effectiveness of the proposed prandial BG control algorithm, we perform BG control simulations in MAT-

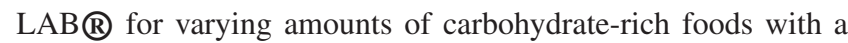
wide range of GI (values in parenthesis); instant potato (83), white bread (71), spaghetti (41) and pearled barley (25). First, for the nominal case, the BG control algorithm is assessed for carbohydrate loads of $50 \mathrm{~g}$ and $100 \mathrm{~g}$ with exact model-patient match and mealtime in the T1D patient model at $k_{\text {food }}=0$. Second, intra-patient variability due to continuous subcutaneous same-tissue utilization [22] and differences in reproducibility of the GI value of a meal [23] are also considered, incorporating $\pm 20 \%$ variation in insulin-dependent glucose uptake (i.e., insulin sensitivity), parameter $p_{3}$ of the T1D patient model, and GI value of the food as given in Eqs. (1) and (14) in the supplementary material. Lastly, food-related uncertainties commonly found in real-life scenarios include misestimation of carbohydrate intake of $\pm 10 \mathrm{~g}$ (20\%) from the nominal amount of $50 \mathrm{~g}$ and deviation in mealtime $\left(k_{\text {food }}= \pm 15 \mathrm{~min}\right)$ in the continuous-time T1D patient model, considering that the assumed mealtime in the controller remains fixed at 0 . Initial parameter values for all simulations are set at fasting state with basal insulin infusion to maintain BG levels at $100 \mathrm{mg} / \mathrm{dL}$. In particular, the function 'fmincon' is utilized exclusively for computation of the cost function in Eq. (3).

\section{Results}

Results of the three aforementioned cases examined in the simulation study are presented in the following subsections.

\subsection{Nominal case}

We assess the performance of the present BG control algorithm for $50 \mathrm{~g}$ of the aforementioned carbohydrate-rich foods with GI values as shown in Fig. 2. The optimal preprandial bolus and postprandial basal insulin infusions are shown separately in Figs. 3 and 4. In particular, Fig. 3 shows that the optimal preprandial bolus insulin follows a hill-shaped infusion pattern with

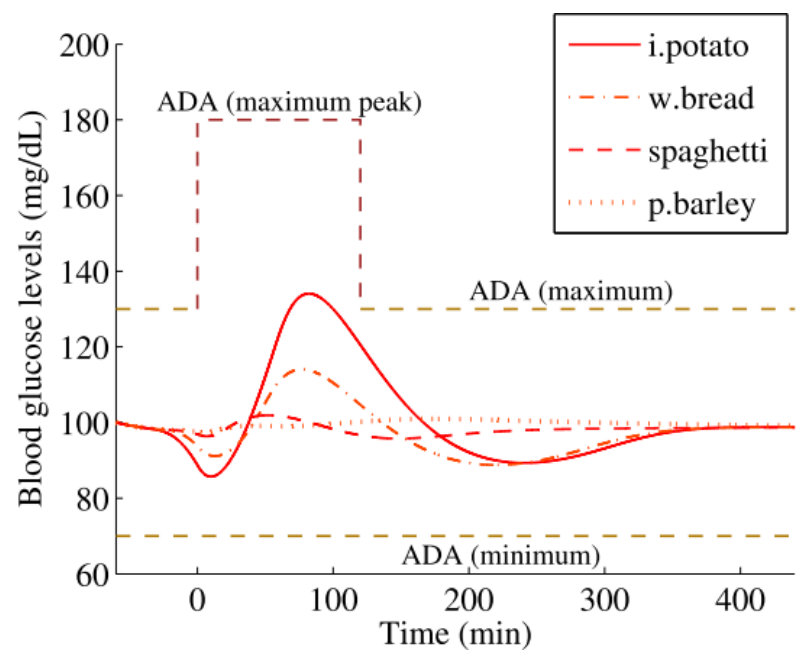

Fig. 2 Prandial BG control response for a meal with carbohydrate content of $50 \mathrm{~g}$ and varying GI (value in parenthesis); instant potato (83), white bread (71), spaghetti (41) and pearled barley (25), consumed at $k_{\text {food }}=0$.

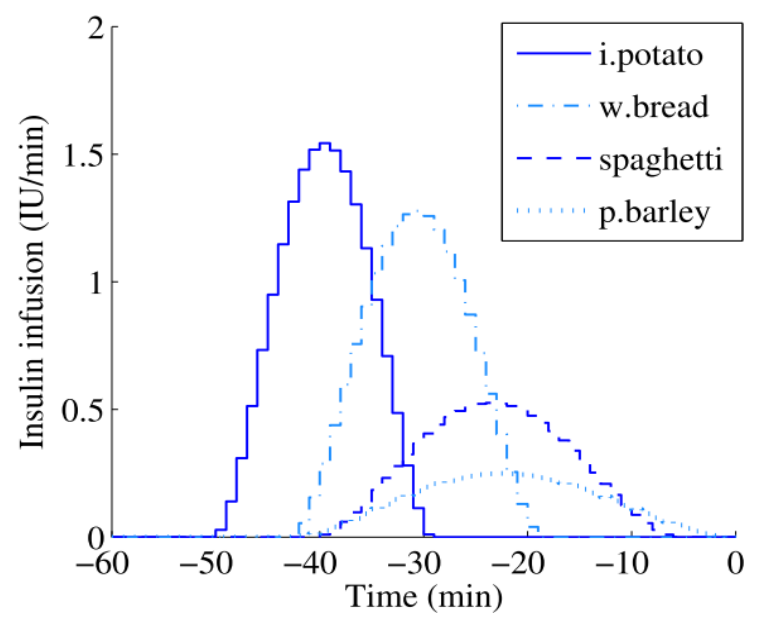

Fig. 3 Optimal preprandial subcutaneous insulin infusion from $k=$ $-60 \mathrm{~min}$ to 0 for a nominal meal containing $50 \mathrm{~g}$ of carbohydrate-rich food (GI value in parenthesis); instant potato (83), white bread (71), spaghetti (41), and pearled barley (25). 


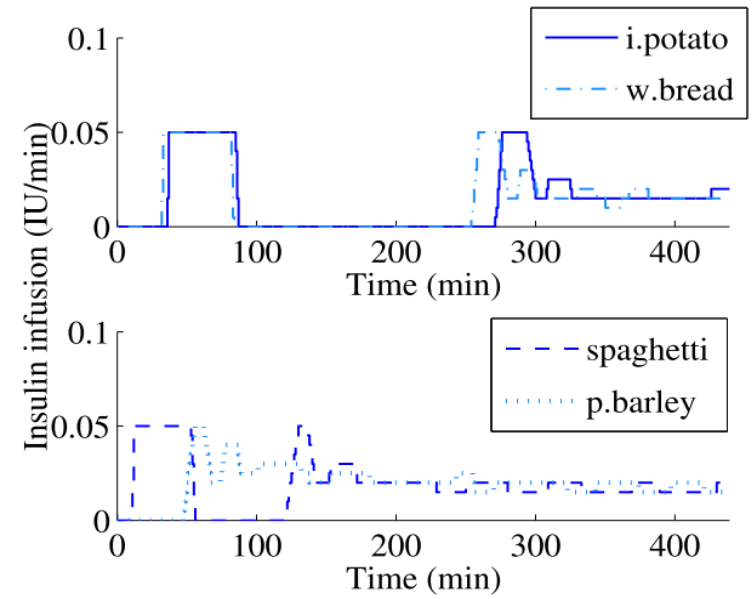

Fig. 4 Closed-loop MPC postprandial subcutaneous insulin infusion from $k=0$ to 440 for a nominal meal of $50 \mathrm{~g}$ of carbohydrate-rich food (GI value in parenthesis): (Top) instant potato (83) and white bread (71); (Bottom) spaghetti (41), and pearled barley (25).

large differences in peak bolus insulin infusion and timing among the representative carbohydrate-rich foods. Note that only the optimal preprandial insulin from $k_{i}$ to 0 is infused to the T1D patient, although the optimization in Eq. (1) computes the total prandial insulin requirement from $k_{i}$ through $k_{f}$. For exact comparison, the range in BG excursion for each type of food is also given in Table 2 . There are definite differences in BG control response between high and low GI foods. BG is maintained close to ideal values for foods with lower GI values, whereas postprandial glycemia is observed with peaks within the ADA recommended value for foods with higher GI values. Additionally, we examine the response of the prandial BG control algorithm for a larger carbohydrate load of $100 \mathrm{~g}$, and the prandial BG control ranges are shown in Table 2. For each food, BG levels are also maintained within similar values as those for a 50-g load with the same GI value, with equally similar insulin infusion pattern depending on the GI value.

\subsection{Intra-individual variability}

Simulation results considering intra-individual variation of parameter $p_{3} \pm 20 \%$ and GI $\pm 20 \%$ in the patient model for a high GI food (instant potato) are shown in Fig. 5. The results indicate that BG levels can be effectively maintained within the ADA recommended values. Slower meal-derived glucose appearance from low GI foods contributes to less pronounced BG excursions directly proportional to the GI value of the food (Table 2).

\subsection{Food-related uncertainties}

Simulation results of food-related uncertainties in mealtime and carbohydrate estimation for a high GI food (instant potato) are shown in Fig. 6, and the BG control ranges for the four representative foods are given in Table 2. Even though postprandial glycemic excursion is maintained below $160 \mathrm{mg} / \mathrm{dL}(8.89 \mathrm{mmol} / \mathrm{L})$ in all cases, there is a risk of borderline hypoglycemia with BG level reaching $75 \mathrm{mg} / \mathrm{dL}$ ( $4.17 \mathrm{mmol} / \mathrm{L}$ ) between 20-30 min due to delayed mealtime, and $79 \mathrm{mg} / \mathrm{dL}(4.39 \mathrm{mmol} / \mathrm{L})$ between 190-220 min caused by early mealtime combined with a 10-g un-
Table 2 Ranges of prandial BG excursion using the present control algorithm for nominal intake of $50 \mathrm{~g}$ and $100 \mathrm{~g}$, with intra-individual variability ( $p_{3}$ and GI value), and with meal-related uncertainties in carbohydrate intake and mealtime.

\begin{tabular}{|c|c|c|}
\hline Simulation case & GI & $\begin{array}{l}\text { BG range } \\
{[\mathrm{mg} / \mathrm{dL}]}\end{array}$ \\
\hline & 83 & 86-134 \\
\hline $50 \mathrm{~g}$ & 71 & $91-114$ \\
\hline \multirow[t]{3}{*}{ (nominal) } & 41 & $96-102$ \\
\hline & 25 & $97-101$ \\
\hline & 83 & $86-152$ \\
\hline $100 \mathrm{~g}$ & 71 & $92-126$ \\
\hline \multirow[t]{3}{*}{ (nominal) } & 41 & 96-104 \\
\hline & 25 & $97-101$ \\
\hline & 83 & $81-157$ \\
\hline$p_{3} \pm 20 \%$ & 71 & $82-135$ \\
\hline \multirow[t]{3}{*}{$50 \mathrm{~g}$} & 41 & $89-112$ \\
\hline & 25 & $92-108$ \\
\hline & 83 & $86-158$ \\
\hline $\mathrm{GI} \pm 20 \%$ & 71 & $87-132$ \\
\hline \multirow[t]{3}{*}{$50 \mathrm{~g}$} & 41 & $93-108$ \\
\hline & 25 & $97-103$ \\
\hline & 83 & $79-159$ \\
\hline$k_{\text {food }}=-15 \min$ & 71 & $81-139$ \\
\hline \multirow[t]{3}{*}{$50 \mathrm{~g} \pm 10 \mathrm{~g}$} & 41 & $93-114$ \\
\hline & 25 & $98-104$ \\
\hline & 83 & $75-148$ \\
\hline$k_{\mathrm{food}}=+15 \mathrm{~min}$ & 71 & $82-122$ \\
\hline \multirow[t]{2}{*}{$50 \mathrm{~g} \pm 10 \mathrm{~g}$} & 41 & $92-106$ \\
\hline & 25 & 94-103 \\
\hline
\end{tabular}

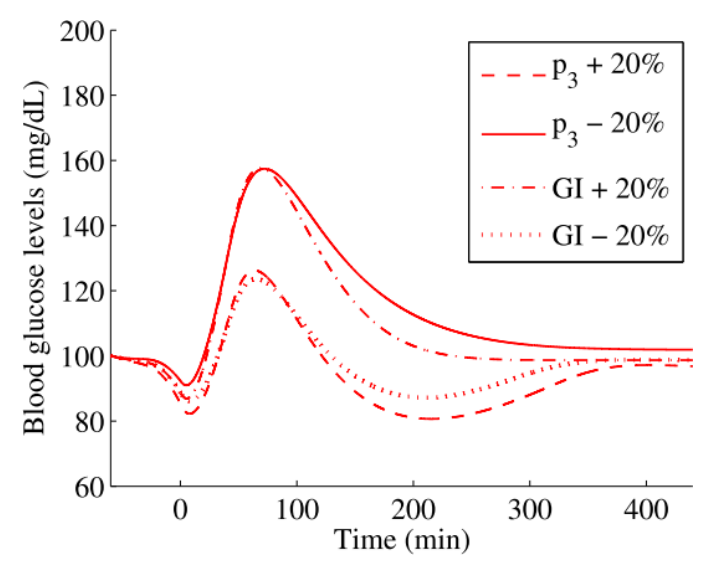

Fig. 5 Simulation of prandial BG control for $50 \mathrm{~g}$ of instant potato (high GI) consumed at $k_{\text {food }}=0$ with intra-individual variability in glucose effectiveness $\left(p_{3} \pm 20 \%\right)$ and GI $\pm 20 \%$.

derestimation of carbohydrate intake.

\section{Discussion}

This study presents an in silico evaluation of a semi closed-loop BG control algorithm for prandial state with prior meal announcement, which consists of preprandial insulin bolus infusion com- 


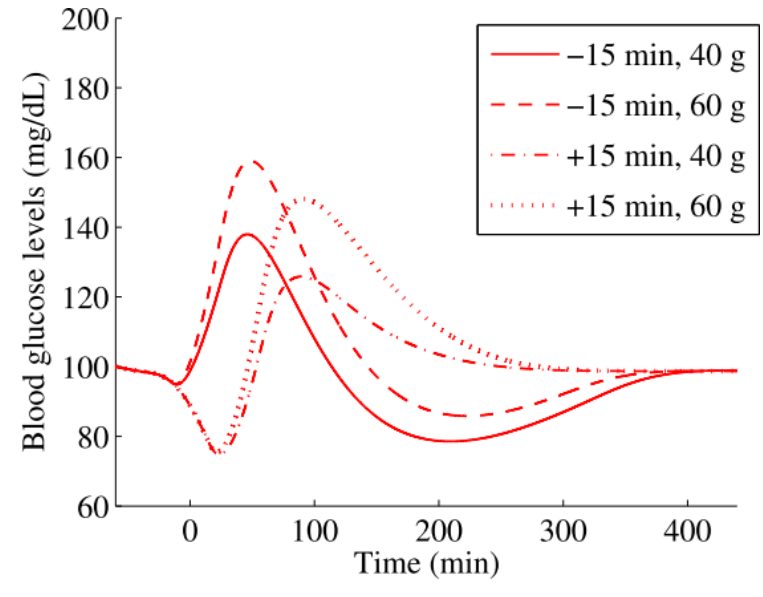

Fig. 6 Simulation of prandial BG control for instant potato (high GI) considering mealtime deviation of $k_{\text {food }}=-15 \min$ (advance) or $k_{\text {food }}=+15 \min$ (delay) in combination with carbohydrate miscalculation of $\pm 10 \mathrm{~g}$ from the intended nominal intake of $50 \mathrm{~g}$.

puted 60 min prior to the intended mealtime, followed by postprandial closed-loop control to maintain BG levels at the target value of $100 \mathrm{mg} / \mathrm{dL}$ as recommended by the ADA. Compared to our previous work [14] which was limited to an instantaneous single-bolus administered at a specific instant between $k_{i}=-30$ and 0 (i.e., single-bolus administration at a specific instant between $30 \mathrm{~min}$ prior to mealtime and $0 \mathrm{~min}$ ), the present study considers continuous variable insulin infusion instead and the potential of starting insulin infusion from $k_{i}=-60$ in order to obtain the optimal preprandial bolus dose and timing for each specific type of food. This study also extends the time span in the cost function to include the total prandial state from $k_{i}$ to $k_{f}$. Moreover, the mathematical model utilized is an enhanced version of the model employed in our previous study [14]. It includes the maximum rate of glucose appearance from carbohydrates [16] to provide a more accurate model-based prediction of postprandial BG excursion in the present study. For a more thorough assessment of the performance of the BG control algorithm, we not only consider the nominal case as in our previous study [14] but also examine variability in subcutaneous insulin absorption as well as uncertainties in food amount and mealtime in the T1D patient model. Considering that daytime hypoglycemia (BG levels below $60 \mathrm{mg}$ / $\mathrm{dL}$ ) in T1D patients has a negative impact on the quality of life and health care cost even for mild episodes [24], the above improvements are necessary to prevent the risk of late postprandial hypoglycemia due to the remnant hypoglycemic effect of insulin several hours after large preprandial bolus doses. In addition, computation of maximum doses for preprandial and postprandial insulin infusions ( $U_{\text {pre }}=2 \mathrm{IU} / \mathrm{min}$ and $\left.U_{\text {post }}=0.05 \mathrm{IU} / \mathrm{min}\right)$ are necessary for safety reasons. In particular, $U_{\text {post }}$ is noticeably above the maximum basal insulin infusion in closed-loop clinical trials [25]. Yet, this dose is considered necessary since slow digestion and absorption of low GI foods require greater insulin infusion rates than the basal rate for as long as 200 min post-meal. Note that the utilization of $U_{\text {pre }}$ and $U_{\text {post }}$ does not affect noticeably the performance of the control algorithm for any of the simulations cases examined in this study.

To our knowledge, the present study demonstrates for the first time the feasibility of tight prandial BG control in patients with T1D even beyond ADA recommendations, although at the expense of starting prandial insulin infusion approximately $50 \mathrm{~min}$ before meal, which is arguably not a practical value for the operation of an artificial pancreas system in everyday conditions. Furthermore, the post-meal glycemic excursions in the simulation results, which are proportional to the GI values of the foods, are identical to those observed in nondiabetics for similar GI values [26]. This finding indicates that the BG control results obtained in the present study are comparable to the ideal response in healthy subjects. Nevertheless, the apparent inability of the present control algorithm to obtain a completely flat BG response for high GI foods-as represented by instant potato-compared to medium and low GI foods actually demonstrates the extent at which tight BG control is feasible under ideal conditions. Moreover, the insulin infusion patterns for nominal carbohydrate intakes of $50 \mathrm{~g}$ and $100 \mathrm{~g}$ indicate that the GI of the food is more relevant than the total intake in determining the infusion pattern, whereas carbohydrate intake only impacts the preprandial insulin dosage. This is consistent with reports from recent clinical studies that emphasize the difficulty in determining an appropriate bolus infusion pattern for a specific meal [27] despite the availability of insulin pump technologies with several bolus infusion patterns. It is also interesting that despite all the foods having an identical carbohydrate content of $50 \mathrm{~g}$, the start of preprandial insulin infusion and the total bolus required differ proportionally to the GI value (Fig. 3), which is supports prior clinical studies [28] suggesting that the current insulin bolus estimation from carbohydrate intake alone should be extended to include the GI value.

Assessment of the present control algorithm beyond the nominal case includes patient-model mismatch (individual variability) and food-related uncertainties. In particular, since parameters of the mathematical model of glucose-insulin metabolism in T1D are assumed to have a good fit for a representative patient, only intra-patient variability is considered as a source of model mismatch. Simulation results not only show that the control algorithm effectively maintains BG levels within the ADA recommended clinical values, but also that spontaneous patient variability regarding insulin sensitivity and glycemic impact of the food, as well as food-related uncertainties in both amount and timing can be managed within a BG peak below $160 \mathrm{mg} / \mathrm{dL}$ $(8.89 \mathrm{mmol} / \mathrm{L})$ and a nadir of $75 \mathrm{mg} / \mathrm{dL}(4.16 \mathrm{mmol} / \mathrm{L})$ in all cases. Although the duration of meal consumption-and consequently impact on BG levels-in the T1D patient model might vary among individuals, the mathematical model previously developed (supplementary material) has been validated by clinical studies in which patients explicitly consumed the meal within $10 \mathrm{~min}$. Hence, differences in postprandial BG excursion due to the meal consumption time are considered to be less relevant than intra-individual variability and food-related uncertainties examined in the present study for assessing the performance of the BG control algorithm.

Previous studies employing bi-hormonal closed-loop BG control systems with both insulin and glucagon infusions [29, 30] utilize the latter to prevent postprandial hypoglycemia, although such approach is known to increase both the complexity of the control system and burden to the patient due to the requirement of additional glucagon infusion. Compared to this, the control algorithm proposed in the present study considers the glycemic im- 
pact of different types of carbohydrates-as represented by their GI values-for more accurate $\mathrm{BG}$ prediction and early insulin infusion as a countermeasure against the well-known delay in subcutaneous absorption. As a result of this 'proactive' approach, the maintenance of BG levels above $75 \mathrm{mg} / \mathrm{dL}$ (Table 2) suggests that an additional algorithm for glucagon infusion might not be necessary for successful diurnal BG control. It should be noted, though, that even with such prediction capabilities, the present control algorithm does not consider insulin-on-board, i.e., residual insulin active for as long as 4-5 hours in the case of rapid-acting insulin, and thus entails a risk of postprandial hypoglycemia in the case of successive meals with few hours in-between. Although the cost function includes a penalty term $Q_{\mathrm{LO}}$ to reduce the risk of hypoglycemia, we believe that the BG impact from successive meals and the overlapped effect of the respective prandial insulin infusions might require more meticulous consideration of the control algorithm law, as well as the case of unplanned additional food or nibbling through a longer time span as in typical real-life scenarios. Nevertheless, due to the satisfactory in silico results achieved in the present study, further clinical implementation might be eventually considered, which would provide more strict evaluations of hypoglycemic risk for patient safety and methods to curtail the time of preprandial insulin infusion for practical reasons,.

\section{Conclusion}

In the present study, the feasibility of a BG control algorithm that maintains tight $\mathrm{BG}$ control during prandial state even beyond ADA recommendations was demonstrated in silico. The total carbohydrate intake and GI value of the food are used in the computation of the optimal prandial insulin infusion under an ideal setting. Tight postprandial BG control is achieved by insulin infusion from 60 min prior to the intended mealtime in combination with closed-loop postprandial BG control based on MPC. Simulation results of the control algorithm proposed in this study show satisfactory performance not only for nominal carbohydrate loads of $50 \mathrm{~g}$ and $100 \mathrm{~g}$ but also with considerations of intra-individual variability and uncertainties in actual carbohydrate intake and mealtime.

\section{Acknowledgement}

This work was funded partially by Grant-in-Aid for Scientific Research (C) (KAKENHI) from the Japan Society for the Promotion of Science (\#26420414, E. Furutani).

\section{Conflict of Interest}

The authors declare no conflicts of interest regarding this manuscript, nor do they have any relationship with companies or commercial organizations based on the definition of Japanese Society of Medical and Biological Engineering.

\section{References}

1. King $\mathrm{AB}$, Armstrong $\mathrm{DU}$ : A prospective evaluation of insulin dosing recommendations in patients with type 1 diabetes at near normal glucose control: bolus dosing. J Diabetes Sci Technol. 1(1), pp. 42-46, 2007.

2. Forst T, Forst S, Strunk K, Löbig M, Welter K, Kazda C, Pfützner A: Impact of insulin on microvascular blood flow and endothelial cell function in the postprandial state in patients with type 1 dia- betes. J Diabetes Complicat. 19(3), pp. 128-132, 2005.

3. Rendell MS, Jovanovic L: Targeting postprandial hyperglycemia. Metab Clin Exp. 55(9), pp. 1263-1281, 2006.

4. Peyser T, Dassau E, Breton M, Skyler JS: The artificial pancreas: current status and future prospects in the management of diabetes. Ann N Y Acad Sci. 1311, pp. 102-123, 2014.

5. Steil GM: Algorithms for a closed-loop artificial pancreas: the case for proportional-integral-derivative control. J Diabetes Sci Technol. 7(6), pp. 1621-1631, 2013.

6. Bequette BW: Algorithms for a closed-loop artificial pancreas: the case for model predictive control. J Diabetes Sci Technol. 7(6), pp. 1632-1643, 2013.

7. Dassau E, Bequette BW, Buckingham BA, Doyle FJ: Detection of a meal using continuous glucose monitoring: implications for an artificial beta-cell. Diabetes Care. 31(2), pp. 295-300, 2008.

8. Weinzimer SA, Steil GM, Swan KL, Dziura J, Kurtz N, Tamborlane WV: Fully automated closed-loop insulin delivery versus semiautomated hybrid control in pediatric patients with type 1 diabetes using an artificial pancreas. Diabetes Care. 31(5), pp. 934-939, 2008.

9. Steil GM, Palerm CC, Kurtz N, Voskanyan G, Roy A, Paz S, Kandeel FR: The effect of insulin feedback on closed loop glucose control. J Clin Endocrinol Metab. 96(5), pp. 1402-1408, 2011.

10. Chase HP, Doyle FJ, Zisser H, Renard E, Nimri R, Cobelli C et al.: Multicenter closed-loop/hybrid meal bolus insulin delivery with type 1 diabetes. Diabetes Technol Ther. 16(10), pp. 623632, 2014.

11. Huyett LM, Dassau E, Zisser HC, Doyle FJ: Design and Evaluation of a Robust PID Controller for a Fully Implantable Artificial Pancreas. Ind Eng Chem Res. 54(42), pp. 10311-10321, 2015

12. American Diabetes Association: Standards of medical care in diabetes-2014. Diabetes Care. 37(Suppl 1), pp. S14-80, 2014.

13. Luijf YM, van Bon AC, Hoekstra JB, Devries JH: Premeal injection of rapid-acting insulin reduces postprandial glycemic excursions in type 1 diabetes. Diabetes Care. 33(10), pp. 2152-2155, 2010

14. Hashimoto S, Yamamoto Noguchi CC, Furutani E: Postprandial blood glucose control in type 1 diabetes for carbohydrates with varying glycemic index foods. Proc. of IEEE EMBC. pp. 48354838, 2014

15. Yamamoto Noguchi CC, Furutani E, Sumi S: Mathematical model of glucose-insulin metabolism in type 1 diabetes including digestion and absorption of carbohydrates. SICE JCMSI. 7(6), pp. 314-320, 2014.

16. Yamamoto Noguchi CC, Hashimoto S, Furutani E, Sumi S: Model of gut absorption from carbohydrates with maximum rate of exogenous glucose appearance in type 1 diabetes. SICE JCMSI. 9(5), pp. 201-206,2016.

17. Jenkins DJ, Wolever TM, Taylor RH, Barker H, Fielden H, Baldwin JM, Bowling AC, Newman HC, Jenkins AL, Goff DV: Glycemic index of foods: a physiological basis for carbohydrate exchange. Am J Clin Nutr. 34(3), pp. 362-366, 1981.

18. Weinstein RL, Schwartz SL, Brazg RL, Bugler JR, Peyser TA, McGarraugh GV: Accuracy of the 5-day FreeStyle Navigator Continuous Glucose Monitoring System: comparison with frequent laboratory reference measurements. Diabetes Care. 30(5), pp. 1125-1130, 2007.

19. Smart CE, Ross K, Edge JA, Collins CE, Colyvas K, King BR: Children and adolescents on intensive insulin therapy maintain postprandial glycaemic control without precise carbohydrate counting. Diabet Med. 26(3), pp. 279-285, 2009. 
20. Scheiner G, Boyer BA: Characteristics of basal insulin requirements by age and gender in type-1 diabetes patients using insulin pump therapy. Diabetes Res Clin Pract. 69(1), pp. 14-21, 2005.

21. Furutani E, Miyake Y, Hagiwara T, Araki M: A design method of robust model algorithmic control systems. 7th SICE Symposium on Control Engineering, pp. 385-388, 1999.

22. Swan KL, Dziura JD, Steil GM, Voskanyan GR, Sikes KA, Steffen AT, Martin ML, Tamborlane WV, Weinzimer SA: Effect of age of infusion site and type of rapid-acting analog on pharmacodynamic parameters of insulin boluses in youth with type 1 diabetes receiving insulin pump therapy. Diabetes Care. 32(2), pp. 240-244, 2009.

23. Vega-López S, Ausman LM, Griffith JL, Lichtenstein AH: Interindividual variability and intra-individual reproducibility of glycemic index values for commercial white bread. Diabetes Care. 30(6), pp. 1412-1417, 2007

24. Brod M, Christensen T, Thomsen TL, Bushnell DM: The impact of non-severe hypoglycemic events on work productivity and diabetes management. Value Health. 14(5), pp. 665-671, 2011.

25. Elleri D, Allen JM, Kumareswaran K, Leelarathna L, Nodale M, Caldwell K, Cheng P, Kollman C, Haidar A, Murphy HR, Wilinska ME, Acerini CL, Dunger DB, Hovorka R: Closed-loop basal insulin delivery over 36 hours in adolescents with type 1 diabetes: randomized clinical trial. Diabetes Care. 36(4), pp. 838-844, 2013.

26. Brand-Miller JC, Stockmann K, Atkinson F, Petocz P, Denyer G: Glycemic index, postprandial glycemia, and the shape of the curve in healthy subjects: analysis of a database of more than 1,000 foods. Am J Clin Nutr. 89(1), pp. 97-105, 2009.

27. López P, Smart C, Morbey C, McElduff P, Paterson M, King BR: Extended insulin boluses cannot control postprandial glycemia as well as a standard bolus in children and adults using insulin pump therapy. BMJ Open Diabetes Res Care. 14(2), pp. e000050, 2014.

28. Parillo M, Annuzzi G, Rivellese AA, Bozzetto L, Alessandrini R, Riccardi G, Capaldo B: Effects of meals with different glycaemic index on postprandial blood glucose response in patients with Type 1 diabetes treated with continuous subcutaneous insulin infusion. Diabet Med. 28(2), pp. 227-229, 2011.

29. Castle JR, Engle JM, El Youssef J, Massoud RG, Yuen KC, Kagan R, Ward WK: Novel use of glucagon in a closed-loop system for prevention of hypoglycemia in type 1 diabetes. Diabetes Care. 33(6), pp. 1282-1287, 2010.

30. Russell SJ, El-Khatib FH, Nathan DM, Damiano ER: Efficacy determinants of subcutaneous microdose glucagon during closed-loop control. J Diabetes Sci Technol. 4(6), pp. 12881304, 2010.

\section{Supplementary Material}

\section{Mathematical Model of glucose-insulin metabolism}

We have proposed a mathematical model of glucose-insulin metabolism in T1D [15,16], which represents a) carbohydrate digestion and absorption, b) subcutaneous insulin pharmacodynamics and c) glucose-insulin metabolism. In particular, carbohydrate digestion and absorption (Fig. S1) consider carbohydrate intake and the GI value to derive the glucose-equivalent amount (GR), and further divided into rapidly (RAG) and slowly (SAG) available glucose according to the Englyst method.

Table S1 lists the variables and parameters of the mathematic model. the main model equations are given by

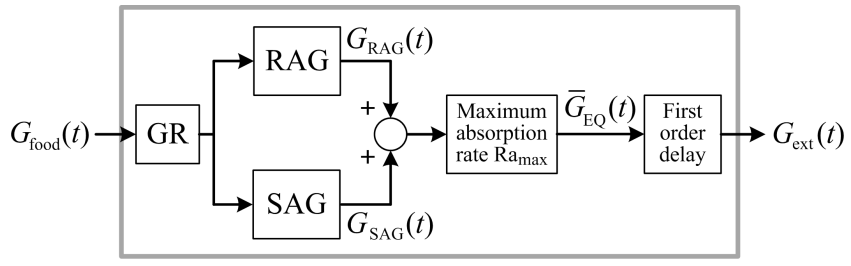

Fig. S1 Diagram of carbohydrate digestion and absorption as glucose-equivalent (GR) amount from carbohydrate intake and GI value, which is further divided into rapidly and slowly available glucose (RAG and SAG). Physiological considerations of maximum absorption rate of meal-derived glucose appearance $\mathrm{Ra}_{\max }$ and delay in gastric emptying (first order delay) are also included.

Table S1 Variables and parameters of the mathematical model of glucose-insulin metabolism from carbohydrates in T1D used in the present study.

$x_{\mathrm{R}}(t) \quad$ State variable for RAG absorption

$x_{\mathrm{S}}(t)$ State variable for SAG absorption

$G_{\text {food }}(t)$ Carbohydrate intake input

$\tau_{\mathrm{dg}}$ Gastric emptying delay constant

$G_{\text {ext }}(t) \quad$ Carbohydrate as glucose-equivalent

$T_{\mathrm{R}}, T_{\mathrm{S}}$ Parameters of RAG and SAG absorption

$k_{\mathrm{R}}, k_{\mathrm{S}} \quad$ Constants of RAG and SAG proportion

GR Glucose equivalent of carbohydrate intake

AvCHO Carbohydrate amount ingested

GI Glycemic index of foods

$k_{1}, k_{2}$ Glucose relative constants

$\bar{G}_{\mathrm{EQ}}(t) \quad$ Glucose-equivalent considering $\mathrm{Ra}_{\max }$

$\mathrm{Ra}_{\max }$ Maximum rate of glucose appearance

$x_{1}(t)$ Insulin mass at the subcutaneous depot

$x_{2}(t)$ Subcutaneous absorption compartment

$I(t)$ Plasma insulin concentration

$V_{\mathrm{d}}$ Plasma distribution volume

$k_{21}$ Insulin diffusion parameter

$k_{\mathrm{a}}$ Insulin transition rate

$k_{\mathrm{d}}$ Degradation rate constant

$k_{\mathrm{e}}$ Degradation rate constant in plasma

$u_{\mathrm{s}}(t) \quad$ Subcutaneous insulin administration

$G(t)$ Blood glucose concentration

$V_{1} \quad$ Volume distribution space

$p_{1}$ Glucose mass action rate constant

$G_{\mathrm{b}} \quad$ Basal blood glucose level

$X(t)$ Remote-insulin concentration

$p_{2}$ Rate of decrease in glucose uptake ability

$p_{3}$ Rate of insulin-dependent glucose uptake ability 


$$
\frac{\mathrm{d}}{\mathrm{d} t}\left[\begin{array}{c}
x_{\mathrm{R}} \\
x_{\mathrm{S}}
\end{array}\right]=\left[\begin{array}{cc}
A_{\mathrm{R}} & 0 \\
0 & A_{\mathrm{S}}
\end{array}\right]\left[\begin{array}{c}
x_{\mathrm{R}} \\
x_{\mathrm{S}}
\end{array}\right]+\left[\begin{array}{c}
B_{\mathrm{R}} \\
B_{\mathrm{S}}
\end{array}\right] G_{\text {food }}(t)
$$

where $G_{\text {food }}(t)$ is the meal input signal of the model, $t_{\text {food }}$ is the total food ingestion period, and further matrix representation of RAG and SAG absorption is given by

$$
\begin{aligned}
& A_{\mathrm{R}}=\left[\begin{array}{cc}
0 & 1 \\
-\frac{1}{T_{\mathrm{R}}^{2}} & -\frac{2}{T_{\mathrm{R}}}
\end{array}\right], \quad A_{\mathrm{S}}=\left[\begin{array}{cc}
0 & 1 \\
-\frac{1}{T_{\mathrm{S}}^{2}} & -\frac{2}{T_{\mathrm{S}}}
\end{array}\right] \\
& B_{\mathrm{R}}=\left[\begin{array}{ll}
0 & \frac{k_{\mathrm{R}}}{T_{\mathrm{R}}^{2}} \mathrm{GR}
\end{array}\right]^{\mathrm{T}}, B_{\mathrm{S}}\left[\begin{array}{ll}
0 & \frac{k_{\mathrm{S}}}{T_{\mathrm{S}}^{2}} \mathrm{GR}
\end{array}\right]^{\mathrm{T}} \\
& G_{\mathrm{RAG}}(t)=C_{\mathrm{R}} x_{\mathrm{R}}(t) \\
& G_{\mathrm{SAG}}(t)=C_{\mathrm{S}} x_{\mathrm{S}}\left(t-\tau_{\mathrm{SAG}}\right)
\end{aligned}
$$

$G_{\mathrm{RAG}}(\mathrm{t})$ and $G_{\mathrm{SAG}}(\mathrm{t})$ represent RAG and SAG absorption, respectively, with $C_{\mathrm{R}}=C_{\mathrm{S}}=\left[\begin{array}{ll}1 & 0\end{array}\right]$, and $\tau_{\mathrm{SAG}}=20 \mathrm{~min}$ for delay of SAG absorption by definition. Maximum rate of meal-derived exogenous glucose appearance $\left(\mathrm{Ra}_{\max }\right)$ followed by food-specific delay in gastric emptying $\left[G_{\text {ext }}(t)\right]$ are also considered as

$$
\begin{aligned}
& \bar{G}_{\mathrm{EQ}}(t)= \begin{cases}G_{\mathrm{RAG}}(t), & t<t_{1} \\
\operatorname{Ra}_{\mathrm{max}}, & t_{1} \leq t \leq t_{2} \\
G_{\mathrm{RAG}}\left(t-t_{3}\right)+G_{\mathrm{SAG}}(t), & t>t_{2}\end{cases} \\
& \frac{\mathrm{d} G_{\mathrm{ext}}(t)}{\mathrm{d} t}=\frac{1}{\tau_{\mathrm{dg}}}\left(-G_{\mathrm{ext}}(t)+\bar{G}_{\mathrm{EQ}}(t)\right)
\end{aligned}
$$

with time parameters $t_{1}, t_{2}$ and $t_{3}$ being specific to the amount and GI value of the food.

Subcutaneous rapid-acting insulin absorption kinetics from Shimoda et al. [5] is given by

$$
\begin{aligned}
& \frac{\mathrm{d} x_{1}(t)}{\mathrm{d} t}=-k_{21} x_{1}(t)+u_{\mathrm{s}}(t) \\
& \frac{\mathrm{d} x_{2}(t)}{\mathrm{d} t}=k_{21} x_{1}(t)-\left(k_{\mathrm{d}}+k_{\mathrm{a}}\right) x_{2}(t) \\
& \frac{\mathrm{d} I(t)}{\mathrm{d} t}=\frac{k_{\mathrm{a}}}{V_{\mathrm{d}}} x_{2}(t)-k_{\mathrm{e}} I(t)
\end{aligned}
$$

Lastly, the glucose-insulin metabolism subsystem from Bergman minimal model [6] comprises

$$
\begin{aligned}
\frac{\mathrm{d} G(t)}{\mathrm{d} t} & =-X(t) G(t)+p_{1}\left(G_{\mathrm{b}}-G(t)\right)+\frac{G_{\text {ext }}(t)}{V_{1}} \\
\frac{\mathrm{d} X(t)}{\mathrm{d} t} & =-p_{2} X(t)+p_{3} I(t)
\end{aligned}
$$

Parameter values of a representative patient with T1D, as well as carbohydrate absorption parameters for four representative staple foods are further detailed elsewhere $[15,16]$.

\section{Claudia Cecilia Yамамото NoguchI}

Claudia Cecilia Yамамото Noguchi received her bachelor's degree from Pontificia Universidad Católica del Perú, Lima, Peru in 2006, and her master's and Ph.D. from Kyoto University, Japan in 2013 and 2016, respectively. She is currently a research student in the same university. Her re-

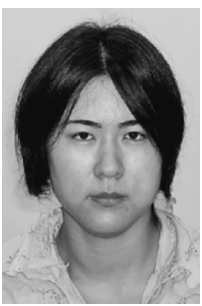
search interests include mathematical modeling of postprandial glucose-insulin metabolism and blood glucose control algorithms for prandial state, both particularly in type 1 diabetes.

\section{Shogo НаSнiмoтo}

Shogo Налнімото received his bachelor's and master's degrees from Kyoto University, Japan, in 2014 and 2016, respectively. He is currently working at Murata Machinery, Ltd. His research interests include design of circuit systems and image processing.

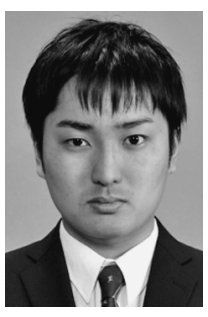

\section{Eiko Furutani}

Eiko FURUTANI received his bachelor's, master's and $\mathrm{Ph} . \mathrm{D}$. degrees from Kyoto University, Japan, in 1987, 1989, and 1997, respectively. In 1991, he joined Kyoto University, where he is currently an Associate Professor of the Department of Electrical Engineering. His research interests include

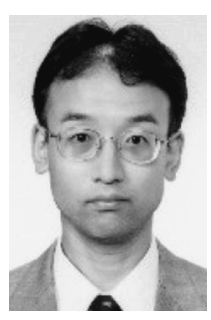
control technologies and their application to medical problems in cooperation with medical doctors. He is a member of IEEE. 